Proceedings

\title{
Geographical accessibility by bus to the University Hospital of Cáceres (Spain) by means of GIS
}

\author{
Montaña Jiménez-Espada ${ }^{1}$
}

1 Department of Construction, School of Technology, University of Extremadura, Avda de la Universidad s/n, CP-10003 Cáceres, Spain

* Correspondence: mjespada@unex.es; Tel.: +34-927251618

Citation: Jimenez, M., 2022, Geographical accessibility by bus to the University Hospital of Cáceres (Spain) by means of GIS. SUPTM 2022 conference proceedings sciforum054548.

https://doi.org/10.31428/10317/10496

Publisher's Note: UPCT and Sciforum stays neutral with regard to jurisdictional claims in published maps and institutional affiliations.

Copyright: (c) 2022 by the authors. Submitted for possible open access publication under the terms and conditions of the Creative Commons Attribution (CC BY) license (https://creativecommons.org/license s/by/4.0/).

\begin{abstract}
Improving the supply and accessibility of public services and facilities in order to satisfy the basic daily needs of the population is one of the premises to be taken into account in the spatial planning of a city. These aspects directly affect the decision-making process in terms of governance and management at the municipal level. In this sense, this research aims to quantify the degree of territorial accessibility available to residents of the city of Cáceres to basic health facilities using public transport, focusing on the recent opening of the new University Hospital on the outskirts of the city and the consequent creation of specific bus lines that provide the transfer service.

For the development of this work, the following elements have been analysed: a) the different routes available by public transport, $b$ ) the set of bus stops present in the city and their distribution within the urban area, c) the characteristics of the potential users of the hospital by establishing age groups and d) the relationship between its initial location (the doorways of the different homes) and the point of access to the public transport service. From the methodological point of view, geographic information systems have been selected as a computer tool for the development of the scientific process (due to their analytical potential), given that they allow the measurement of geographic accessibility by means of applications based on network analysis. The visualization of the information on the accessibility results obtained is carried out by means of raster cartographic representation. Considering the minimum access time to the hospital as a factor of analysis, there are notable differences between different neighborhoods in the city. However, when subdivided into time slots, no substantial differences can be observed. On the other hand, a higher population density is clearly observed in the inner-city districts.
\end{abstract}

Keywords: Territorial accessibility, GIS, public transport, urban facilities, spatial analysis

\section{Introduction}

The rapid and disorganized growth of cities, together with the absence in previous decades of long-term urban planning, poses a risk in the location of public services, particularly hospitals, since in certain cases today they are located in the centre of cities, and therefore they face the problem of not being resilient (capable of adapting, expanding and serving the aforementioned increase in population). Faced with this situation, the need arises to locate newly built hospitals on the outskirts of cities (Guerras, 2003). In these new locations, the aim is to favour and guarantee access to these services for users residing in the city, without these displacements being at the cost of an increase in the use of private vehicles. It is therefore necessary to have a public transport service that could be able to adapt to the new demand caused by the change of location, ensuring accessibility to it (Fernández, 2009).

While public transport plays an important role in the overall mobility structure of large cities, in small and medium-sized cities, similar in size to Cáceres, the structure of passenger distribution between the different means of transport is altered, with private 
transport gaining special importance, as opposed to the limited supply of public transport (Pérez and López, 2001).

In recent years there has been growing interest in the study of the specific problems of mobility and accessibility in small to medium sized historic towns. Providing mobility to the population, while at the same time making it compatible with the preservation of the town's urban environment and its historical heritage has become a difficult challenge. In a World Heritage city such as Cáceres, the constant increase in mobility has caused certain circulation, parking, noise, and pollution problems which have affected negatively both the population's quality of life and the historical heritage.

In the case of the city of Cáceres, the peculiar distribution of the different areas of the municipality makes good public transport planning particularly complicated. The situation of the old town represents the extreme end of the spectrum on the mobility and accessibility problems of the city, while the progressive population increase in the peripheral areas and its exclusive focus on the use of private vehicles, highlight the poor mobility alternatives (Jiménez-Espada, 2016).

The aim of this study is to analyze the accessibility by public transport to the University Hospital of Cáceres. To achieve this purpose, it is intended to use geographic information systems tools. In order to understand this accessibility in a clear way, an analysis of it is carried out from the following points of view:

- Description of the accessibility of the different neighbourhoods of the city with respect to the selected focus of study (University Hospital). To this end, the population density of each one is related to its level of accessibility, allowing a comparison to be made between them.

- Existing variation in accessibility depending on the time of day and the availability of the different urban bus lines.

- Identification of the possible differences in accessibility that may appear within the same neighbourhood, due to the distribution of stops and lines that provide service.

Finally, the aim is to understand the use that users currently make of the L3 bus line, which provides access to the University Hospital, in order to identify inefficiencies and possible solutions for improvement that could be undertaken

\section{Methodology}

For the development of this study, we worked with data in different formats and from different sources.

Regarding the modelling with GIS software, the information was obtained mainly through the Spatial Data Infrastructure (SDI) of the Geographic Information Service of the City Council of Cáceres.

Since the focus of the study is on the urban bus service, and in order to characterize the different public transport operating lines, it was decided to obtain information on the time taken to travel on each line, taking into account the travel time between stops. For this purpose, several sources were consulted, as shown below, in order to have a wide range of data and to be able to contrast them with each other.

Firstly, we used the real-time positioning service available on Cáceres city buses. This functionality is offered through the portal http://opendata.caceres.es/. On the other hand, the same calculation of times between stops was carried out based on the data offered by the concessionary company of the service, SUBUS, on its own web page https://caceres.vectalia.es/. In order to complete and contrast the data obtained from the previous sources, the concessionary company SUBUS provided access to its facilities and its fleet management system, obtaining information on the theoretical travel times of the different lines, real times, theoretical/actual differences and the percentage of punctuality.

The first step carried out when approaching this study was planning. To this end, we proceeded to plan and identify the different elements that would make up the accessibility calculation. 
We proceeded to create a structure for the displacements (figure 1), establishing the origin and destination of the same, as well as the stops to be used, both for access and transfer. At the same time, the different bus lines were characterized according to the time spent on their journeys.

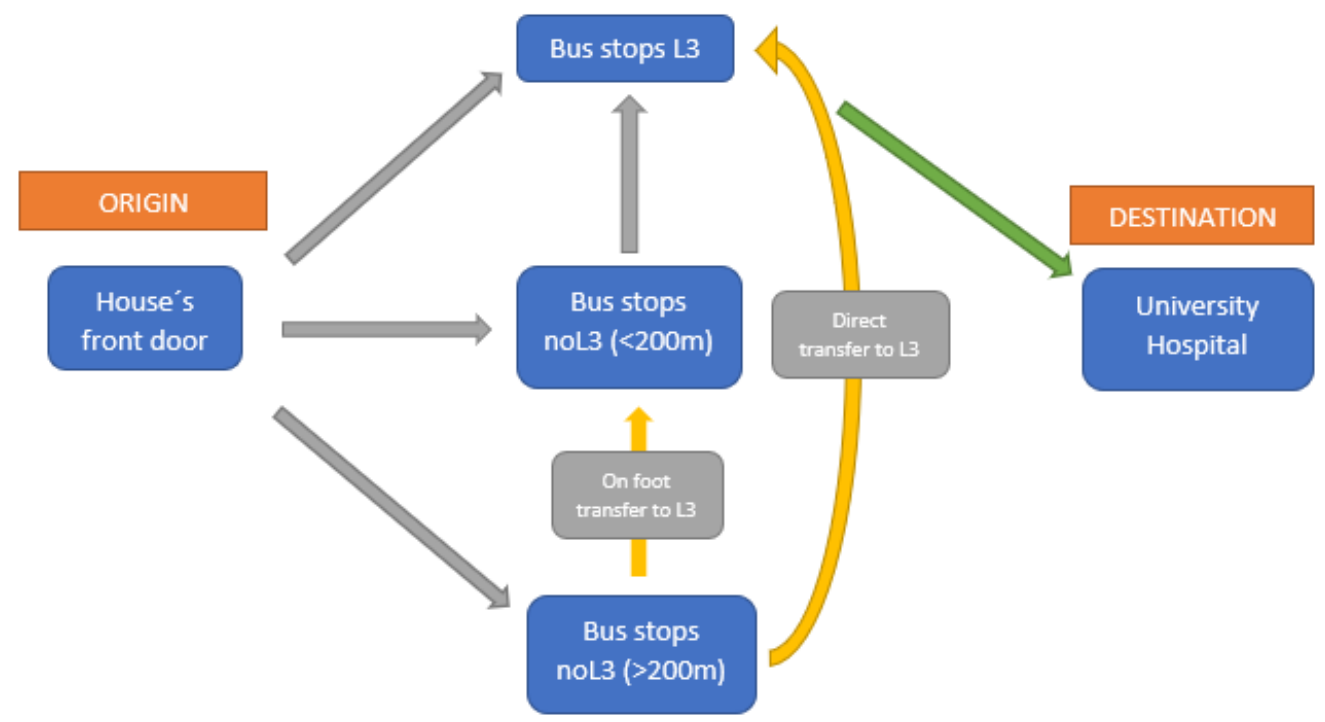

Figure 1. Structure for the displacements.

In the case of the choice of bus stops, it was taken into account that this selection by users in order to access the public transport service would not only be influenced by the shortest distance, but also by other variables involved in the final decision (Sánchez Fernández et al, 2017).

In the preparation of the study, it was observed that transfers from other lines to the line that connects to the Hospital could be made at stops on the line itself that corresponds to the Hospital line, or at a stop that, although it does not correspond to the Hospital line, is within an acceptable walking distance of a stop on the Hospital line itself.

Finally, three different time slots were established in which accessibility to the hospital was analysed. These time slots differ from each other in the variation of lines available in each one.

\section{Results}

From the point of view of access times, considering the time slots considered, it was observed that the best accessibility situation is obtained in the morning. This is due to the configuration of the line during this shift. Due to the existence of a greater availability of stops that favour a better connection with the line to the Hospital and, therefore, a reduction in access time. In the remaining scenarios, no significant differences in access times were observed.

When analysing the access times according to the different neighbourhoods in the city, it was observed that those that presented a more favourable situation were those located in the eastern part of the city. The central and central-northern areas presented reduced access times, while the neighbourhoods where the greatest deficiencies were detected were those located in the western and southern areas of the city centre.

In order to facilitate the interpretation of the routes obtained in the results, it was decided to use a graphic representation of an eminently visual and intuitive nature 
(figure 2). In this way, the starting neighbourhood, the route to follow and the time needed to reach the hospital are defined.

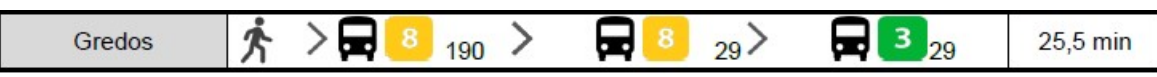

Figure 2. Routes graphic representation.

\section{Conclusions}

Taking into account the results obtained in the present study, it is concluded that:

- Regarding accessibility, there are notable differences between the different districts of the city, from the point of view of the minimum access time to the University Hospital.

- In general, there are no differences in accessibility depending on the time slot considered, except in those neighbourhoods located in the northern part of the city where a significant improvement is observed in the morning time slots.

- No significant time differences are estimated between the different portals located within the same neighbourhood, which shows a correct distribution of the stops within them.

- There is a higher population density in the neighbourhoods located in the city centre, areas where the level of accessibility is adequate.

According to the results obtained in this study, it can be concluded that, although the urban transport service of the city of Cáceres covers efficiently the extension of its territory, there are significant differences between different areas of the city in terms of total travel times.

When compared to cities with similar characteristics to Cáceres, it is worth highlighting the need for a joint approach to solve the traffic problem in these medium-sized cities, with the aim of bringing together both the planning of the road network and its management, as well as intermodality between the different modes of transport. Specifically, it is necessary to offer quality and coordinated public transport, which is presented as a revulsive in the face of a private traffic that is practiced in conditions of collapse, and levels of terrible service of the collector roads of the populations.

\section{References}

1. Fernández, L. (2009). ¿Podemos diseñar y construir hospitales más eficientes en el futuro? XVI Congreso Nacional de Hospitales. Extremadura (España).

2. Guerras, J. J. S. (2003). Verticalidad versus horizontalidad. Historia de la construcción de hospitales en el siglo XX. Informes de la Construcción, vol. 55, no 485, p. 13-25.

3. Jiménez-Espada, M., González-Escobar, R. (2016). Comments and Suggestions for Improvement of Mobility and Road Safety in the City of Cáceres. Procedia engineering, vol. 161, p. 1385-1390.

4. Pérez, I., López Fontán, S. (2001). La gestión de la red viaria en ciudades de tamaño medio. In IRF Global Road Congress (14‥ 2001. París). International Road Federation (IRF).

5. Sánchez Fernández, M., Fragoso Campón, L., Gutiérrez Gallego J. A., Quirós Rosado E. (2017): “Caída de la demanda con la distancia en el uso del transporte público: el caso del acceso al campus universitario de Cáceres", GeoFocus (Artículos), no 20, p. 141-163. 